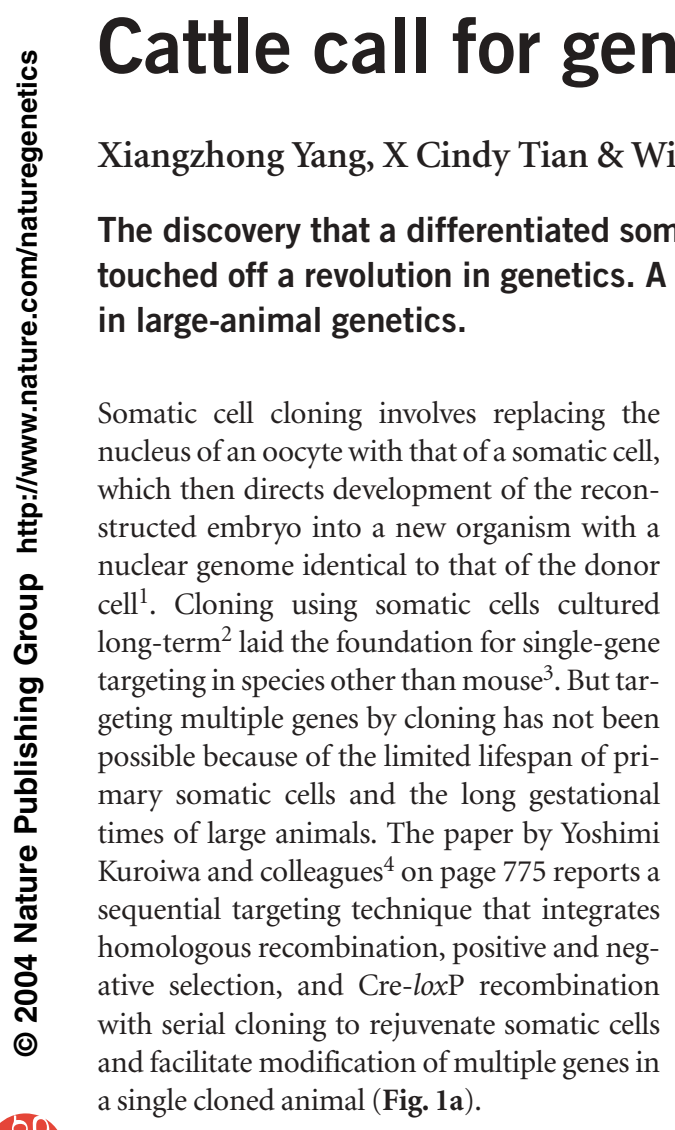

\section{Rejuvenation by serial cloning}

Generating a homozygous single-gene knockout cow would take $\sim 6$ years using conventional methods (Fig. 1b). First, a gene of interest would be knocked out by homologous recombination in cultured somatic cells (typically fibroblasts), which would then be cloned to create a heterozygous calf with a targeted mutation. The heterozygotes would be raised to maturity for breeding ( $\sim 2$ years of age) to generate first-generation $\left(\mathrm{F}_{1}\right)$ male and female heterozygous calves, which would be crossbred to generate a homozygous knockout calf. Generating homozygotes with respect to multiple targeted mutations using the conventional approach in cattle would be impractical.

In contrast, the sequential targeting strategy

Xiangzhong Yang, X. Cindy Tian and William Fodor are at the Center for Regenerative Biology, University of Connecticut, 1392 Storrs Road, Storrs, Connecticut 06269, USA. described by Kuroiwa et al. makes multiple genetic modifications in cattle, allowing the generation of homozygous cows in less than 2 years (Fig. 1a). First, the targeted cloned embryos are transferred to foster mothers to generate fetuses (not for term development, but for rejuvenating somatic cells for the next round of gene targeting). The rejuvenated fetal cells are then collected and subjected to a second round of homologous recombination

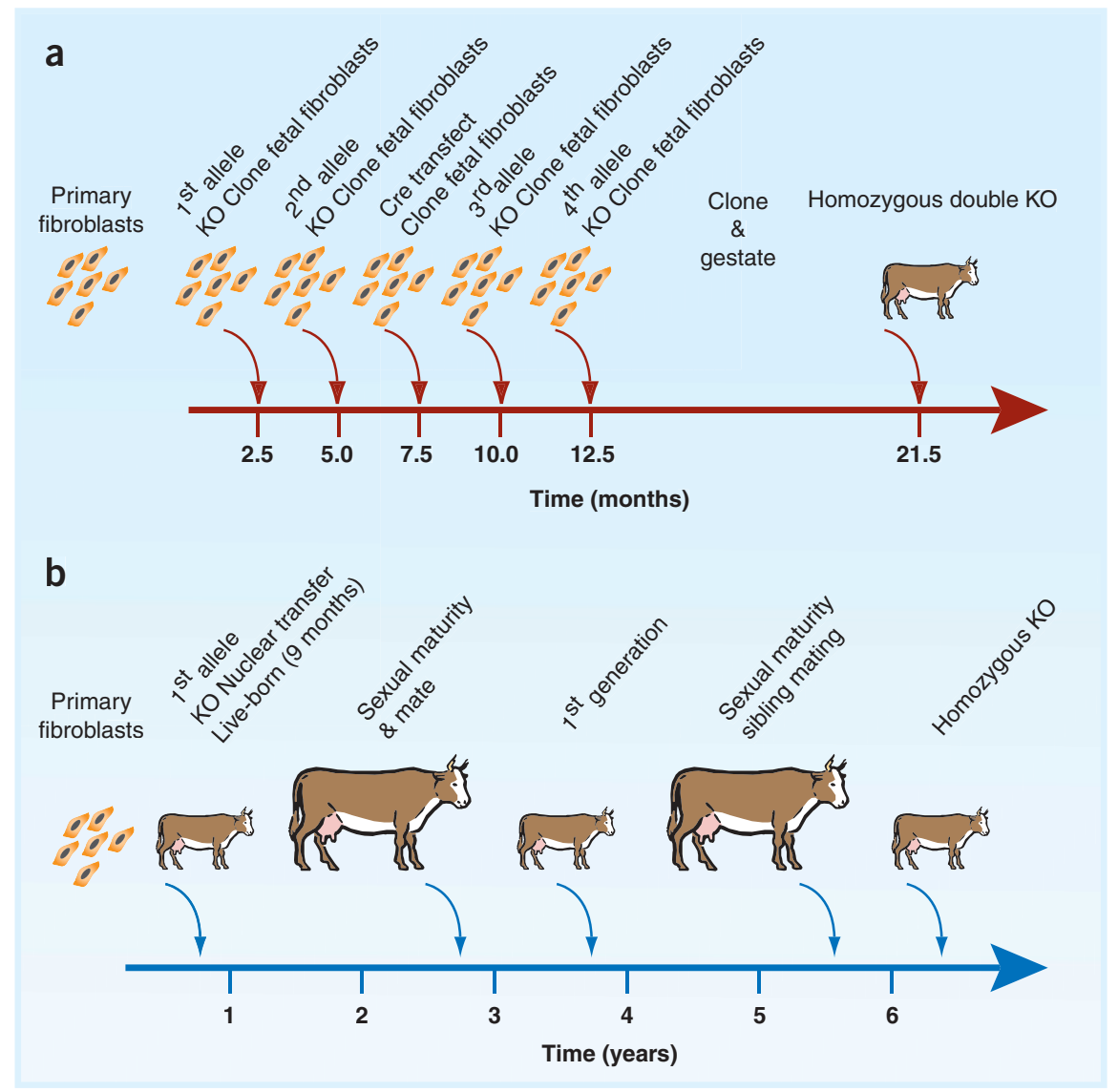

Figure 1 Rapid generation of homozygous double-knockout livestock. (a) Transfection of fibroblasts with a targeted gene knockout (KO) construct, followed by nuclear transfer, cloning to rejuvenate new primary fibroblasts and subsequent rounds of transfection and cloning, yields double-knockout cells from which a cloned cow can be derived in less than 2 years. (b) Generating homozygous single-gene knockout (KO) cows by conventional means would take $\sim 5$ years from the birth of the first cloned cows. 
to target the second allele, thereby generating homozygous knockout fetuses. Using this approach, each targeting event requires only 2.5 months, and a calf homozygous with respect to two targeted genes can be produced in less than 24 months (Fig. 1a).

The third and fourth rounds of marker selections present a challenge for sequentially targeting a second gene. To circumvent this problem, the authors recycled the same selectable mark$\mathrm{ers}^{5}, n e o^{\mathrm{r}}$ and puro $^{\mathrm{r}}$, in their sequential gene targeting efforts, using the Cre-lox $\mathrm{P}$ system to remove the drug resistance markers used in the first and second rounds of gene targeting. This allows them to use limited selection markers to target multiple genes in a single animal. The $n e o^{\mathrm{r}}$ and puro $^{\mathrm{r}}$ marker genes were not expressed in the cloned fetuses, probably owing to methylation of these genes in the donor cells during cloning reprogramming, as transgenes are often silenced by DNA methylation in transgenic animals ${ }^{6}$. Whether silencing of the marker genes will be a persistent feature of cloning or a random inactivation phenomenon has yet to be determined. The finding that the Cre recombinase plasmid integrated into the genome in most fetuses was also unexpected, but this problem might be solved with further technical improvements to the methodology.

Primary somatic cells have a finite lifespan in culture and would probably become senescent after one or two rounds of gene targeting. Kuroiwa et al. used serial cloning to rejuvenate primary somatic cells in order to accomplish multiple rounds of gene targeting. Cell rejuvenation in clones by rebuilding of eroded telomeres from aged donor cells in cattle $^{7,8}$ has been confirmed previously. Telomere reprogramming has also been found after serial cloning in cattle ${ }^{9}$ and mice ${ }^{10}$. Notably, the pregnancy success rate after four rounds of fetal serial cloning was as high as $71 \%$; however, the efficiency for term development is not yet known. The discrepancy between this study and previous serial cloning reports ${ }^{9,10}$, which found progressive decreases in cloning efficiency, is probably due to the different source of donor cells (adult versus fetal cells).

\section{Bigger, better, faster}

Sequential targeting will undoubtedly be used to produce valuable livestock and create cattle resistant to deadly diseases, such as bovine spongiform encephalopathy (BSE). BSE is believed to be caused by prions, aberrant forms of a normal prion protein, PrP. Approximately 200,000 cattle have been diagnosed with $\mathrm{BSE}^{11}$ and more than 130 people have died from BSE ${ }^{12}$ since the first outbreak in 1996. This and previous studies ${ }^{11,12}$ indicate that PRNP may be knocked out to create cows resistant to BSE. Although certain lines of PrP knockout mice are healthy, the health of PrP knockout cattle and the effectiveness of making such cattle resistant to BSE infection have not yet been tested.

Another benefit of mutating PRNP applies to using cattle as bioreactors. Medical products such as human immunoglobulin and human serum albumin produced in transgenic cattle must be quality-controlled to be prion-free. Mutating PRNP in these cattle could help ensure that bovine-derived recombinant protein products are free of prions.

Another area of livestock research that will benefit from the technique is xenotransplantation. The demand for organs suitable for human transplantation far exceeds the current donor supply. Research in the area of xenotransplantation has focused on the development of transgenic pigs that express human genes ${ }^{13}$ or carry targeted mutations in detrimental genes ${ }^{14}$. The ability to create multiple genetic modifications could be used to disrupt active porcine endogenous retroviral sequences in the pig genome and eliminate cross-species retroviral infection (xenozoonosis). The potential to insert beneficial genes, mutate detrimental genes and eliminate the potential for xenozoonosis by sequential cloning are just a few of the potential applications of rapid, serial targeting.

Finally, sequential cloning in livestock may also benefit the development of largeanimal models of human disease in areas where rodent models prove difficult to create or are ineffective at mimicking human disease phenotypes ${ }^{15}$.

1. Wilmut, I., Schnieke, A.E., McWhir, J. Kind, A.J. \& Campbell, K.H. Nature 338, 810-813 (1997).

2. Kubota, C. et al. Proc. Natl. Acad. Sci. USA 97, 990-995 (2000)

3. Capecchi, M.R. Proc. Natl. Acad. Sci. USA 97 , 956-957 (2000).

4. Kuroiwa, Y. et al. Nat. Genet. 36, 775-780 (2004).

5. Abuin, A. \& Bradley, A. Mol. Cell. Biol. 16, 1851-1856 (1996).

6. Feng, Y.Q., Lorincz, M.C., Fiering, S., Greally, J.M. \& Bouhassira, E.E. Mol. Cell. Biol. 21, 298-309 (2001).

7. Tian, X.C., Xu, J. \& Yang, X. Nat. Genet. 26, 272-273 (2000).

8. Lanza, R.P. et al. Science 288, 665-669 (2000).

9. Kubota, C., Tian, X.C. \& Yang, X. Nat. Biotechnol. 22, 693-694 (2004).

10. Wakayama, T. et al. Nature 407, 318-319 (2000).

11. Normile, D. Science 303, 1285 (2004).

12. Cyranoski, D. Nature 426, 743 (2003).

13. Costa, C. et al. Xenotransplantation 9, 45-57 (2002)

14. Phelps, C.J. et al. Science 299, 411-414 (2003).

15. Hardcastle, J., Harwood, M.D. \& Taylor, C.J. J. Pharm. Pharmacol. 56, 329-338 (2004).

\title{
Aging counts on chromosomes
}

\author{
Oscar Fernandez-Capetillo \& André Nussenzweig
}

\section{The acquisition of an abnormal number of chromosomes is a hallmark of many human cancers. A new study indicates that unequal segregation of genetic material to daughter cells during cell division can also lead to premature senescence and accelerated onset of a variety of aging phenotypes.}

The French comedian Maurice Chevalier once said that "growing old isn't so bad when

Oscar Fernandez-Capetillo and André

Nussenzweig are in the Experimental

Immunology Branch, National Cancer Institute,

National Institutes of Health, Bethesda,

Maryland 20892, USA. e-mail:

andre_nussenzweig@nih.gov you consider the alternative." It is becoming clear that the accumulation of DNA damage has a key role in age-related cellular degeneration. Normal aging itself is associated with increased mutation rates and genomic instability, which may also contribute to the increased cancer risk that comes with age ${ }^{1,2}$. Furthermore, the inactivation of certain DNA repair pathways increases the rate at which aging phenotypes develop ${ }^{1}$. At the cellular level, one hallmark of these 'accelerated' aging models is the increase in various types of chromosomal abnormalities, including mutations, translocations, fusions and fragmented chromosomes. Damaged chromosomes are detected by a protein network that triggers either cell death (apoptosis) or a permanent arrest of cell division (senescence), 